\title{
Identification de la prednisone et de la méthylprednisolone dans les cheveux par HPLC-ES-MS
}

\section{Identification of prednisone and methylprednisolone in human hair by $H P L C-E S-M S$}

\section{Vincent CIRIMELE ${ }^{(1)}$, Pascal KINTZ(1), Jean-Pierre GOULLÉ( ${ }^{(2)}$, Bertrand LUDES ${ }^{(1)}$}

(1) Institut de Médecine Légale, 11, rue Humann - F-67085 STRASBOURG France (2) Laboratoire de Biochimie - BP 24 - F-76083 LE HAVRE France

(Reçu le 15 juin 2000 ; accepté le 5 septembre 2000)

\section{$R E ́ S U M E ́$}

Cet article décrit une méthode de dosage de la prednisone et de la méthylprednisolone par chromatographie liquide haute performance couplée à la spectrométrie de masse par une interface de type electrospray. Les cheveux ont été prélevés chez 11 patients sous corticothérapie après avoir obtenu leur consentement oral. Dans la majorité des cas, ce traitement faisait suite à une greffe. Les cheveux ont été préalablement lavés par deux bains successifs de dichlorométhane et réduits en poudre dans un broyeur à boulet. La solubilisation des composés a été réalisée par incubation de $100 \mathrm{mg}$ de poudre de cheveux dans du tampon Soerensen (16h à $40^{\circ} \mathrm{C}$ ) en présence de $50 \mathrm{ng}$ de cortisol-d $d_{3}$ utilisé en temps qu'étalon interne. La purification a été réalisée sur cartouches Isolute CI8 suivie d'une extraction liquide-liquide par de l'éther à $\mathrm{pH}$ alcalin. La phase organique finale a été évaporée puis l'extrait sec repris dans $30 \mu l$ de méthanol. La séparation chromatographique des composés a été réalisée sur une colonne Novapack C18 à l'aide d'un gradient d'acétonitrile de 20 à $60 \%$ en 4 min. Le détecteur utilisé était un spectromètre de masse Perkin Elmer Sciex API 100. La réponse du détecteur était linéaire pour des concentrations de prednisone et de méthylprednisolone variant de 20 à $2000 \mathrm{pg} / \mathrm{mg}$. Les rendements d'extraction pour des cheveux surchargés en corticostéroïdes à la concentration finale de $250 \mathrm{pg} / \mathrm{mg}$ étaient de $70 \%$ pour la prednisone et $75 \%$ pour

\section{SUMMARY}

This paper describes a screening procedure based upon high-performance liquid chromatography / electrospray / mass spectrometry for the identification and quantification of prednisone and methylprednisolone in human hair. Hair specimens were obtained from 11 patients under corticosteroids treatment after verbal consent. Hair strands were washed in methylene chloride, pulverized in a ball mill and $100 \mathrm{mg}$ of the powdered hair were incubated in $1 \mathrm{ml}$ Soerensen buffer, pH 7.6 for $16 \mathrm{~h}$ at $40^{\circ} \mathrm{C}$, in presence of $50 \mathrm{ng}$ cortisol- $\mathrm{d}_{3}$ used as internal standard. Purification of the incubation medium was achieved on SPE C18 Isolute extraction columns followed by an alkaline liquid-liquid extraction with diethylether. The eluate was evaporated to dryness and resuspended in $30 \mu$ l of methanol. The chromatography was operated on a Novapak C18 column using a linear gradient of acetonitrile from 20 to $60 \%$ in 4 min. The detector was a Perkin Elmer Sciex API 100 mass spectrometer. The detector response was linear for prednisone and methylprednisolone concentrations ranging from 20 to $2000 \mathrm{pg} / \mathrm{mg}$. Extraction recovery at $250 \mathrm{pg} / \mathrm{mg}$ was $70 \%$ for prednisone and $75 \%$ for methylprednisolone. Repeatability ( $C V$ values) at 250 $\mathrm{pg} / \mathrm{mg}$ were 8 and $12 \%$ for prednisone and methylprednisolone, respectively. The limits of detection, calculated for a signal to noise ratio of 2 , were 15 and $10 \mathrm{pg} / \mathrm{mg}$ for predni- 
la méthylprednisolone. A cette même concentration, la répétabilité intra-jour était de $8 \%$ pour la prednisone et $12 \%$ pour la méthylprednisolone. Les limites de détection, déterminées pour un rapport signal/bruit de fond équivalent à 2 , étaient de $15 \mathrm{pg} / \mathrm{mg}$ pour la prednisone et $10 \mathrm{pg} / \mathrm{mg}$ pour la méthylprednisolone. Lors de cette étude, la prednisone a pu être identifiée dans les cheveux de 9 patients sur 10 traités par Cortancyl (30 à $130 \mathrm{pg} / \mathrm{mg}$, moyenne $65 \mathrm{pg} / \mathrm{mg}$ ). La méthylprednisolone a pu être dosée $(15 \mathrm{pg} / \mathrm{mg})$ dans les cheveux de l'unique patient traité par Médrol ${ }^{\circledR}$.

\section{MOTS-CLÉS}

Corticostéroïdes, cheveux, HPLC/MS, suivi, dopage.

\section{Introduction}

Les corticostéroïdes naturels et de synthèse sont largement utilisés en milieu médical pour leurs propriétés anti-inflammatoires. Ils peuvent être administrés suite à une greffe ou pour traiter une ostéoporose, une néphropathie ou encore une glomérulonéphrite. Lorsqu'ils sont administrés par voie systémique, ils influencent la production naturelle de corticostérö̈des de l'organisme. Différents procédés analytiques ont été publiés à ce jour pour le dosage des corticostéroïdes dans les fluides biologiques comme les tests radio-immunologiques (1), la chromatographie liquide haute performance (HPLC) avec détection spectrophotométrique (2), la chromatographie en phase gazeuse (GC) (3) et la chromatographie (HPLC ou GC) couplée à la spectrométrie de masse (MS) $(4,5)$. Le sang et les urines sont généralement analysés pour déterminer la prise d'un médicament, mais l'élimination de ces substances intervient en quelques heures pour le sang et en quelques jours pour les urines ( 7 jours pour l'acétonide de triamcinolone). Par contre, la recherche au niveau des phanères tels que les cheveux permet de suivre une administration prolongée sur plusieurs mois (en fonction de la longueur de la mèche de cheveux analysée) et donc de mettre en évidence une exposition répétée (6). Ce moyen d'investigation peut se révéler intéressant pour le suivi des patients sous corticothérapie pour deux raisons essentielles : ces substances sont souvent prescrites à long terme pour traiter des maladies chroniques et leurs nombreux effets secondaires conduisent souvent à un mauvais suivi du traitement prescrit.

Dans le monde du sport, l'utilisation des corticostéroïdes pour leurs propriétés anti-inflammatoires et régénératrices des microtraumatismes cellulaires a conduit la commission médicale du Comité International Olympique à les classer dans la liste des substances dopantes soumises à restrictions. En effet, l'utilisation des corticostéroïdes est interdite, excepté pour usage local, par inhalation et par injection intra- sone and methylprednisolone, respectively. In the presented study, prednisone was identified in the hair of $9 / 10$ patients treated by Cortancyl ${ }^{\circledR}$ (30 à $130 \mathrm{pg} / \mathrm{mg}$, mean $65 \mathrm{pg} / \mathrm{mg}$ ). Methylprednisolone was detected (15 pg/mg) in the hair specimen obtained from the unique patient treated with Medrol ${ }^{\circledR}$.

\section{KEY-WORDS}

Corticosteroids, hair, HPLC/MS, monitoring, doping.

articulaire ou locale, à condition de produire une ordonnance médicale avant la compétition (7). Dans le cadre de la protection de la santé des sportifs (suivi longitudinal), l'usage chronique de ces substances immunosuppressives ne peut être mis en évidence par une analyse urinaire. Par contre, le cheveu possède la propriété unique d'être le marqueur des expositions répétées ou chroniques, permettant en outre d'établir le profil de consommation à long terme et son évolution. L'analyse des cheveux permettra d'augmenter la fenêtre de détection des corticostéroïdes et de faire la différence entre usage unique et abus chronique ou de mettre en évidence un dopage hors compétition.

Les premiers travaux concernant le dosage des corticostéroïdes dans les cheveux ont été récemment publiés (8-11). Ces substances rejoignent donc la longue liste des molécules incorporées dans les cheveux, mais le faible nombre d'échantillons positifs recensé ne permet pas de préjuger de la sensibilité des techniques mises au point.

Cette étude rapporte le développement d'une nouvelle méthode pour le dosage de certains corticostéroïdes dans les cheveux.

\section{Matériel et méthodes}

\section{Réactifs}

Le dichlorométhane, l'acétone, l'hexane, l'acétonitrile, l'éther diéthylique et le méthanol $(\mathrm{MeOH})$ étaient de qualité HPLC (Merck, Allemagne). L'acide formique $(\mathrm{HCOOH})$ était de qualité Normatom (Prolabo, France) et le formiate d'ammonium $\left(\mathrm{NH}_{4} \mathrm{COOH}\right)$ de qualité analytique (Fluka, Allemagne).

La prednisolone et la méthylprednisolone ont été achetées chez Sigma (France). L'analogue deutéré du cortisol (cortisol- $\mathrm{d}_{3}$ ) a été acheté chez Cambridge Isotope Laboratories (CIL, USA).

Les colonnes Isolute $\mathrm{C} 18$ provenaient de chez Touzart et Matignon (France). 


\section{Préparation des solutions}

Le tampon Soerensen a été préparé en ajoutant $38,8 \mathrm{ml}$ de tampon $\mathrm{KH}_{2} \mathrm{PO}_{4}(9,07 \mathrm{~g} / \mathrm{l})$ à $61,2 \mathrm{ml}$ de tampon $\mathrm{Na}_{2} \mathrm{HPO}_{4}(11,87 \mathrm{~g} / \mathrm{l})$. La valeur du $\mathrm{pH}$ a été ajustée à 7,6 .

Le tampon de la phase mobile était constitué d'une solution $2 \mathrm{mM}$ de $\mathrm{NH}_{4} \mathrm{COOH}$ ajustée à un $\mathrm{pH}$ de 3 avec $\mathrm{HCOOH}$.

Les solutions de prednisone et de méthylprednisolone ont été préparées aux concentrations finales de 10,1 et $0,1 \mathrm{mg} / \mathrm{L}$ dans du méthanol. La solution méthanolique de cortisol- $\mathrm{d}_{3}$ a été préparée à la concentration finale de $1 \mathrm{mg} / \mathrm{L}$.

\section{Prélèvements}

Les échantillons de cheveux ont été prélevés chez 11 patients sous corticothérapie après avoir obtenu leur consentement oral. Dans la majorité des cas, ce traitement faisait suite à une greffe ou pour traiter une ostéoporose, une néphropathie ou encore une glomérulonéphrite. Pour chacun d'entre eux, une mèche de cheveux de la région occipitale a été cerclée par une cordelette, coupée le plus près possible du cuir chevelu et stockée dans une enveloppe à température ambiante.

Avant l'analyse, les cheveux ont été lavés dans deux bains successifs de dichlorométhane $(5 \mathrm{ml}, 2 \mathrm{~min}$ à température ambiante) puis réduits en poudre dans un broyeur à boulet (Retsch, type MM2, Allemagne).

\section{Extraction}

Dans chaque cas, environ $100 \mathrm{mg}$ de poudre de cheveux ont été incubés dans $1 \mathrm{ml}$ de tampon Soerensen $\left(16 \mathrm{~h}\right.$ à $\left.40^{\circ} \mathrm{C}\right)$ en présence de $50 \mathrm{ng}$ d'étalon interne, le cortisol- $\mathrm{d}_{3}$. La purification des substances a été réalisée sur colonne Isolute $\mathrm{C} 18$ : le milieu d'incubation a été déposé sur les colonnes activées (méthanol et eau bidistillée), puis rincé successivement par $1 \mathrm{ml}$ du mélange acétone/eau bidistillée $(2: 8, \mathrm{v} / \mathrm{v}), 1 \mathrm{ml}$ d'eau bidistillée, et finalement $1 \mathrm{ml}$ d'hexane. Après séchage des colonnes (30 min), les corticostérö̈des ont été élués par 3 volumes $(0,5 \mathrm{ml})$ successifs de $\mathrm{MeOH}$. L'éluat a été évaporé sous azote et le résidu sec repris par $0,5 \mathrm{ml}$ de soude $0,2 \mathrm{~N}$ pour être ré-extrait par $3 \mathrm{ml}$ d'éther diéthylique. Après agitation et centrifugation, la phase organique finale a été évaporée et l'extrait repris par $30 \mu \mathrm{l}$ de $\mathrm{MeOH}$.

\section{Conditions d'analyse}

Une fraction de $2 \mu 1$ a été injectée dans la colonne chromatographique (Waters, 4- $\mu \mathrm{m}$ Novapak C18, 150 × 2 $\mathrm{mm})$ protégée par une pré-colonne $(5 \mu \mathrm{m}$ Opti-Guard
C18, $15 \times 1 \mathrm{~mm}$ ). La séparation a été obtenue à l'aide d'une phase mobile composée d'acétonitrile et de tampon $\mathrm{NH}_{4} \mathrm{COOH} 2 \mathrm{mM}$ pH 3. Le gradient linéaire d'acétonitrile (20 to $60 \%$ en $4 \mathrm{~min}$ ) était généré par une pompe HPLC (Applied Biosystems, Modèle 140B) au débit de $200 \mu \mathrm{l} / \mathrm{min}$. Un diviseur post-colonne (1:3) a permis d'infuser un débit optimal de $50 \mu \mathrm{l} / \mathrm{min}$ au niveau de l'interface.

La détection a été réalisée par un spectromètre de masse Perkin Elmer Sciex API 100. L'azote (degré de pureté $99.95 \%$ ) a été utilisé comme gaz nébuliseur. La tension d'orifice a été optimisée et fixée à la valeur $+20 \mathrm{~V}$ pour la détection des fragments positifs. L'acquisition a été réalisée en mode sélection d'ions $(\mathrm{m} / \mathrm{z}$ : prednisone 359 , méthylprednisolone 375 , cortisol-d $\left.\mathrm{d}_{3} 366\right)$.

\section{Paramètres de validation}

Les courbes de calibration ont été obtenues après extraction, par le procédé établi, de $100 \mathrm{mg}$ de poudre de cheveux témoins (négatifs pour les corticostéroïdes) additionnés de prednisone et de méthylprednisolone aux concentrations finales de $20 ; 50 ; 250 ; 1000$ et $2000 \mathrm{pg} / \mathrm{mg}$. La répétabilité intra-jour $(\mathrm{n}=8)$ et le rendement d'extraction ont été déterminés à la concentration finale de $250 \mathrm{pg} / \mathrm{mg}$ pour les deux corticostéroïdes. Les limites de détection pour les deux molécules ont été obtenues en abaissant leur concentration finale jusqu'à obtenir un rapport signal/bruit de fond égal à 2 .

Tous ces paramètres de validation ont été déterminés après ajout de $50 \mathrm{ng}$ de cortisol- $\mathrm{d}_{3}$ et extraction par le procédé établi.

\section{Résultats}

\section{Validation}

Dans les conditions opératoires décrites, il n'a pas été noté une quelconque interférence des constituants du cheveu avec les substances recherchées ou l'étalon interne. Les chromatogrammes en mode sélection d'ions de la figure 1 ont été obtenus par le même procédé d'extraction et de purification mais avec 2 milieux d'incubation différents. En haut, le cas d'un échantillon de cheveu positif pour la prednisone à la concentration de $1,28 \mathrm{ng} / \mathrm{mg}$ après incubation de la poudre de cheveux homogénéisée dans du tampon Soerensen. En bas, le même échantillon incubé dans du méthanol (prednisone : 0,32 ng/mg). En accord avec les données de la littérature (12), les solutions aqueuses solubilisent les xénobiotiques incorporés dans les cheveux avec un meilleur rendement que les solvants organiques. 


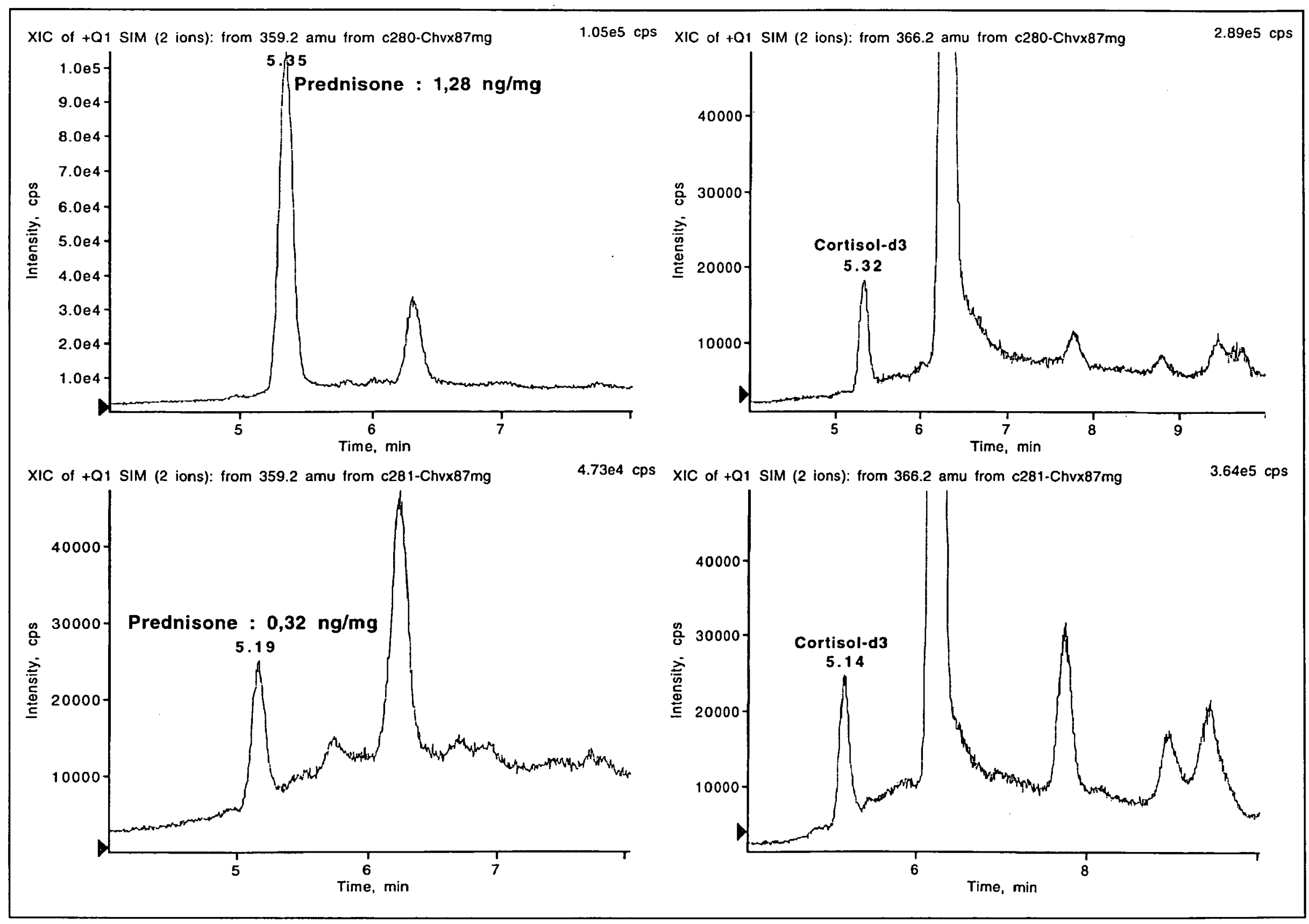

Figure 1 : Chromatogrammes en mode sélection d'ions d'un échantillon de cheveux positif pour la prednisone. En haut, le tracé obtenu après incubation dans le tampon Soerensen et extraction par le procédé décrit. En bas, le tracé obtenu après incubation méthanolique et extraction par le même procédé. Le rendement de solubilisation de la prednisone est 4 fois meilleur dans le tampon que dans le solvant.

Les résultats concernant les paramètres de validation sont donnés dans le tableau I. La réponse du détecteur était linéaire pour des concentrations de prednisone $(\mathrm{r}=0,998)$ et de méthylprednisolone $(\mathrm{r}=0,997)$ variant de 20 à $2000 \mathrm{pg} / \mathrm{mg}$. Les coefficients de variation (répétabilité intra-jour à $250 \mathrm{pg} / \mathrm{mg}$ ) étaient de $8 \%$ pour la prednisone et $12 \%$ pour la méthylprednisolone, avec des rendements d'extraction respectifs de 70 et 75 $\%$. Les limites de détection, déterminées pour un rapport signal/bruit de fond égal à 2 , étaient de $15 \mathrm{pg} / \mathrm{mg}$ pour la prednisone et $10 \mathrm{pg} / \mathrm{mg}$ pour la méthylprednisolone.

\section{Étude clinique}

Les échantillons de cheveux ont été prélevés chez 11 patients sous corticothérapie : dix étaient traités par Cortancyl ${ }^{\circledR}$ (prednisone) et un seul par Médrol ${ }^{\circledR}$ (méthylprednisolone). Les résultats analytiques sont rapportés dans le tableau II. La prednisone a pu être identifiée dans les cheveux de 9 patients sur 10 traités par Cortancyl®. Les concentrations déterminées variaient de 30 à $130 \mathrm{pg} / \mathrm{mg}$ avec une valeur moyenne de $65 \mathrm{pg} / \mathrm{mg}$. Le chromatogramme en ion sélectionné de la figure 2 illustre le cas d'un échantillon de cheveu positif pour la prednisone. La concentration calculée était de $45 \mathrm{pg} / \mathrm{mg}$.

Le seul prélèvement resté négatif avait été prélevé chez une femme traitée par Cortancyl® à la posologie de 5 $\mathrm{mg} / \mathrm{j}$ mais dont les cheveux avaient été colorés. Plusieurs publications rapportent qu'un traitement cosmétique tel que la coloration, la décoloration ou la permanente conduisent à la perte plus ou moins importante du contenu en xénobiotiques des cheveux $(13,14)$.

La méthylprednisolone a pu être dosée à la concentration de $15 \mathrm{pg} / \mathrm{mg}$ dans les cheveux de l'unique patient traité par Médrol ${ }^{\circledR}$ (Tableau II). 
Annales de Toxicologie Analytique, vol. XIII, $\mathrm{n}^{\circ} 1,2001$

Tableau I : Linéarité, rendement d'extraction, répétabilité intra-jour et limite de détection pour la prednisone et la méthylprednisolone après purification par la procédure décrite.

\begin{tabular}{|c|c|c|}
\hline & Prednisone & Méthylprednisolone \\
\hline $\begin{array}{c}\text { Linéarité } \\
(\mathbf{2 0} \text { à } \mathbf{2 0 0 0} \mathbf{~ p g} / \mathbf{m g})\end{array}$ & $\mathrm{r}=0,998$ & $\mathrm{r}=0,997$ \\
\hline $\begin{array}{c}\text { Rendement d'extraction } \\
(\mathbf{2 5 0} \mathbf{~ p g} / \mathbf{m g})\end{array}$ & $70 \%$ & $75 \%$ \\
\hline $\begin{array}{c}\text { Coefficient de variation } \\
(\mathbf{2 5 0} \mathbf{~ p g} / \mathbf{m g})\end{array}$ & $8 \%$ & $12 \%$ \\
\hline $\begin{array}{c}\text { Limite de détection } \\
(\mathbf{S} / \mathbf{N}=\mathbf{2})\end{array}$ & $15 \mathrm{pg} / \mathrm{mg}$ & $10 \mathrm{pg} / \mathrm{mg}$ \\
\hline
\end{tabular}

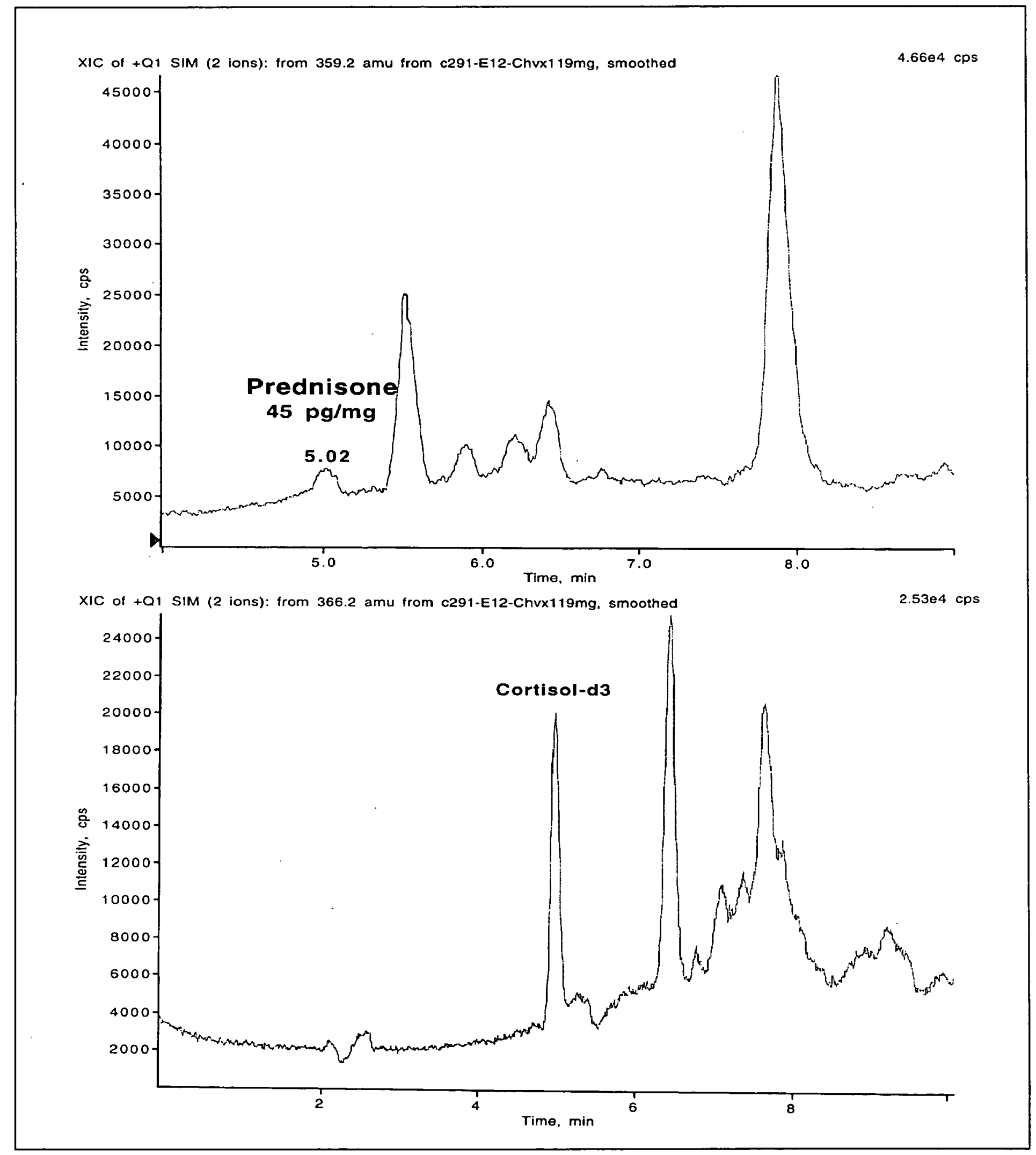

Figure 2 : Chromatogrammes en mode sélection d'ions d'un échantillon de cheveux positif pour la prednisone. La concentration calculée était de $45 \mathrm{pg} / \mathrm{mg}$. 
Tableau II : Résultats des dosages de prednisone et méthylprednisolone chez 11 patients traités par Cortancyl® $(n=10)$ et par Médrol® $(n=1)$.

\begin{tabular}{|c|c|c|}
\hline Patient & Traitement $/$ Posologie & Concentration \\
\hline $\mathbf{1}^{*}$ & Cortancyl $^{\circledR} / 5 \mathrm{mg} / \mathrm{j}$ & Prednisone $<15 \mathrm{pg} / \mathrm{mg}$ \\
\hline $\mathbf{2}$ & Cortancyl $^{\circledR} / 5 \mathrm{mg} / \mathrm{j}$ & Prednisone $30 \mathrm{pg} / \mathrm{mg}$ \\
\hline $\mathbf{3}$ & Cortancyl $^{\circledR} / 5 \mathrm{mg} / \mathrm{j}$ & Prednisone $39 \mathrm{pg} / \mathrm{mg}$ \\
\hline $\mathbf{4}$ & Cortancyl $^{\circledR} / 10 \mathrm{mg} / \mathrm{j}$ & Prednisone $30 \mathrm{pg} / \mathrm{mg}$ \\
\hline $\mathbf{5}$ & Cortancyl $^{\circledR} / 10 \mathrm{mg} / \mathrm{j}$ & Prednisone $57 \mathrm{pg} / \mathrm{mg}$ \\
\hline $\mathbf{6}$ & Cortancyl $^{\circledR} / 20$ à $6 \mathrm{mg} / \mathrm{j}$ & Prednisone $45 \mathrm{pg} / \mathrm{mg}$ \\
\hline $\mathbf{7}$ & Cortancyl $^{\circledR} / 55$ à $9 \mathrm{mg} / \mathrm{j}$ & Prednisone $63 \mathrm{pg} / \mathrm{mg}$ \\
\hline $\mathbf{8}$ & Cortancyl $^{\circledR} / 40$ à $35 \mathrm{mg} / \mathrm{j}$ & Prednisone $103 \mathrm{pg} / \mathrm{mg}$ \\
\hline $\mathbf{9}$ & Cortancyl $^{\circledR} / 50$ à $25 \mathrm{mg} / \mathrm{j}$ & Prednisone $130 \mathrm{pg} / \mathrm{mg}$ \\
\hline $\mathbf{1 0}$ & Cortancyl $^{\circledR} / 60$ à $35 \mathrm{mg} / \mathrm{j}$ & Prednisone $90 \mathrm{pg} / \mathrm{mg}$ \\
\hline $\mathbf{1 1}$ & Médrol $^{\circledR} / 4$ à $2 \mathrm{mg} / \mathrm{j}$ & Méthylprednisone $15 \mathrm{pg} / \mathrm{mg}$ \\
\hline
\end{tabular}

* Patient dont les cheveux étaient colorés

\section{Discussion}

Les concentrations de prednisone détectées lors de cette étude sont inférieures à celle déterminée $(1,28 \mathrm{ng} / \mathrm{mg})$ dans le cadre d'une expertise médicolégale (8). Lors d'une étude préliminaire (9), la recherche de corticostéroïdes par une méthode de screening n'avait permis de recenser que 2 cas positifs sur 6 testés (prednisone $140 \mathrm{pg} / \mathrm{mg}$, béclométhasone $230 \mathrm{pg} / \mathrm{mg}$ ).

Plus récemment, deux études ont été réalisées sur deux groupes de sportifs. Dans le premier cas (10), il s'agissait de 12 cyclistes professionnels pour lesquels la recherche de corticostéroïdes par HPLC/MS/MS s'est avérée positive dans 5 cas avec les concentrations suivantes : triamcinolone acétonide 0,14 à $0,33 \mathrm{ng} / \mathrm{mg}$ $(n=3)$, hydrocortisone acétate $1,31 \mathrm{ng} / \mathrm{mg} \quad(n=1)$, méthylprednisolone $1,21 \mathrm{ng} / \mathrm{mg}(\mathrm{n}=1)$. La deuxième étude (11), réalisée auprès de 19 athlètes, a permis de déterminer l'abus chronique de corticostéroïdes dans 4 cas avec les concentrations suivantes : triamcinolone acétonide $0,28 \mathrm{ng} / \mathrm{mg}(\mathrm{n}=1)$, hydrocortisone acétate $0,43 \mathrm{ng} / \mathrm{mg}(\mathrm{n}=1)$, méthylprednisolone $1,35 \mathrm{ng} / \mathrm{mg}$ $(\mathrm{n}=1)$, béta/dexaméthasone $1,31 \mathrm{ng} / \mathrm{mg}(\mathrm{n}=1)$. Dans ce cas, les analyses étaient réalisées par HPLC/MS.

\section{Conclusion}

En regard des concentrations mesurées dans le cadre de cette étude clinique, le procédé de purification (solubilisation, double extraction sur cartouche et en phase liquide) et les moyens de détection semblent plus satisfaisants que ceux décrits lors de la précédente étude (9). Certes, le nombre d'échantillons testés reste faible $(\mathrm{n}=11)$, mais le fort taux de positivité dans les cheveux (>90\%) atteste de la qualité de la procédure mise en place. La sensibilité analytique est donc d'une importance capitale pour le dosage des corticostéroïdes dans les cheveux.

Après une validation complète de la procédure analytique, la recherche des corticostéroïdes dans les cheveux peut apporter un complément d'informations aux tests conventionnels pratiqués dans le monde du sport (15). Les moyens d'éviter des résultats urinaires positifs pour les corticostéroïdes (prescription médicale) seront inefficaces puisque les cheveux permettront d'établir un profil de consommation de ces substances sur des périodes plus longues, alors que ces substances sont prescrites généralement pour un usage ponctuel. De plus, le prélèvement est simple, moins invasif que le recueil d'urines et peut être conservé à température ambiante. 


\section{Références}

1. Altmeyer P., Buhles N., Hölzel C., Spiteller G., Stöhr L., Holzmann H., Influence of topical corticosteroids and hormones in urine and plasma. Arzneimittelforsch. 1986 ; 36 : 993-6.

2. Jusko W.J., Pyszczynski N.A., Bushway M.S., D'Ambrosio R., Mis S.M., Fifteen years of operation of a high-performance liquid chromatographic assay. J. Chromatogr. B 1994 ; 658 : 47-54.

3. Logunov V.P., Mazklar S.A., Chromatographic measurement of urinary steroids in patients with psoriasis. Klin. Lab. Diagn. 1994 ; 4 : 11-3.

4. Shibasaki H., Furuta T., Kasuya Y., Quantification of corticosteroids in human plasma by liquid chromatographythermospray mass spectrometry using stable isotope dilution. J. Chromatogr. B 1997 ; 692 : 7-14.

5. Delahaut P., Jacquemin P., Colemonts Y., Dubois M., De Graeve J., Deluyker H., Quantitative determination of several synthetic corticosteroids by gas chromatography mass spectrometry. J. Chromatogr. B 1997 ; 696 : 203-15.

6. Drug testing in hair. In P. Kintz (Ed.), CRC Press, Boca Raton, 1996.

7. http://www.nodoping.org/medch2_f.html

8. Cirimele V., Kintz P., Tracqui A., Ludes B., First identification of prednisone in human hair by HPLC-IS/MS. J. Anal. Toxicol. $1999 ; 23: 225-6$.
9. Cirimele V., Kintz P., Dumestre V., Goullé J.P., Ludes B., Identification of ten corticosteroids in human hair by liquid chromatography-ionspray mass spectrometry. Forensic Sci. Int. $2000 ; 107: 381-8$

10. Gaillard Y., Vayssette F., Pépin G., Compared interest between hair analysis and urinalysis in doping controls. Results for amphetamines, corticosteroids and anabolic steroids in racing cyclists. Forensic Sci. Int. 2000 ; 107 : 361-79.

11. Bevalot F., Gaillard Y., Lhermitte M.A., Pépin G., Analysis of corticosteroids in hair by liquid chromatography-electrospray ionisation mass spectrometry. J. Chromatogr. B $2000 ; 740$ : 227-36.

12. Kintz P., Sachs H., Critical review of chromatographic procedures since 1992. J. Chromatogr. B 1998 ; 713 : 147-61.

13. Cirimele V., Kintz P., Mangin P., Drug concentrations in human hair after bleaching. J. Anal. Toxicol. $1995 ; 19$ : 331-2.

14. Yegles M., Marson Y., Wennig R., Influence of bleaching on stability of benzodiazepines in hair. Forensic Sci. Int. $2000 ; 107: 87-92$.

15. Kintz P., Hair testing and doping control in sport. Toxicol. letters $1998 ; 102: 109-13$. 